\title{
OP-Textilien für optimale Rahmenbedingungen
}

\author{
Tragekomfort beeinflusst Konzentration und Reaktionsvermögen
}

Die wichtigste Infektionsquelle ist der Mensch. Maßnahmen zur Infektionsprävention konzentrieren sich daher auf Maßnahmen im Umfeld der medizinischen Akteure. Im hochsensiblen Bereich der Chirurgie stellen höchste Hygienestandards möglichst sichere Bedingungen zur Vermeidung von in vielen Fällen lebensbedrohlichen Infektionen dar. Standardisierte Aufbereitungs- und Sterilisationsverfahren von OP-Bekleidungen und OP-Abdeckungen sind ein wichtiger Baustein im Multibarrierensystem der Infektionsprävention, stellt Dr. Klaus-Dieter Zastrow, Institut für Hygiene und Umweltmedizin, Berlin fest. Die Hygienebedingungen sind für die Entscheidung für Mehrweg- oder Einwegsysteme daher nicht relevant. Der Komfort des Operateurs und seiner Mitarbeiter und die Kosten geben den Ausschlag für die Wahl. Ein Konsensusstatement, das nun präsentiert wurde, hält den State of the Art bezüglich Hygiene, Wirtschaftlichkeit, Tragekomfort und Aufbereitung für Mehrwegtextilien im OP fest.

Bei der Schutzwirkung von genormten OP-Textilien gegen Bakterien und Viren gebe es kaum Unterschiede, erklärte Zastrow. Aufmerksamkeit verdienen vermehrt die thermophysiologischen Eigenschaften von Mehrweg- und Einwegprodukten. „Je besser die thermophysiologischen Eigenschaften sind," so Zastrow, „,desto geringer ist der Temperaturanstieg beim Träger und das verlängert die körperliche Belastbarkeit. Bei ungünstigen thermophysiologischen Eigenschaften kommt es hingegen zu Unwohlsein, Wärmestress und vorzeitigen Konzentrationsmängeln. "Eine Studie des Forschungsinstituts Hohenstein über die Leistungsfähigkeit des OPTeams im Zusammenhang mit der verwendeten OP-Kleidung kommt zum Schluss, dass Mehrweg-OP-Kleidung zu kürzeren Reaktionszeiten auf optische und akustische Reize sowie zu einer geringeren Fehlerquote führen. Vor allem bei längeren oder mehreren aufeinanderfolgenden Operationen gewinnen diese Faktoren an Bedeutung.

„Ohne perfekte Arbeitsbedingungen werden auch keine optimalen Leistungen erbracht", betonte Univ.-Prof. Dr. Harald

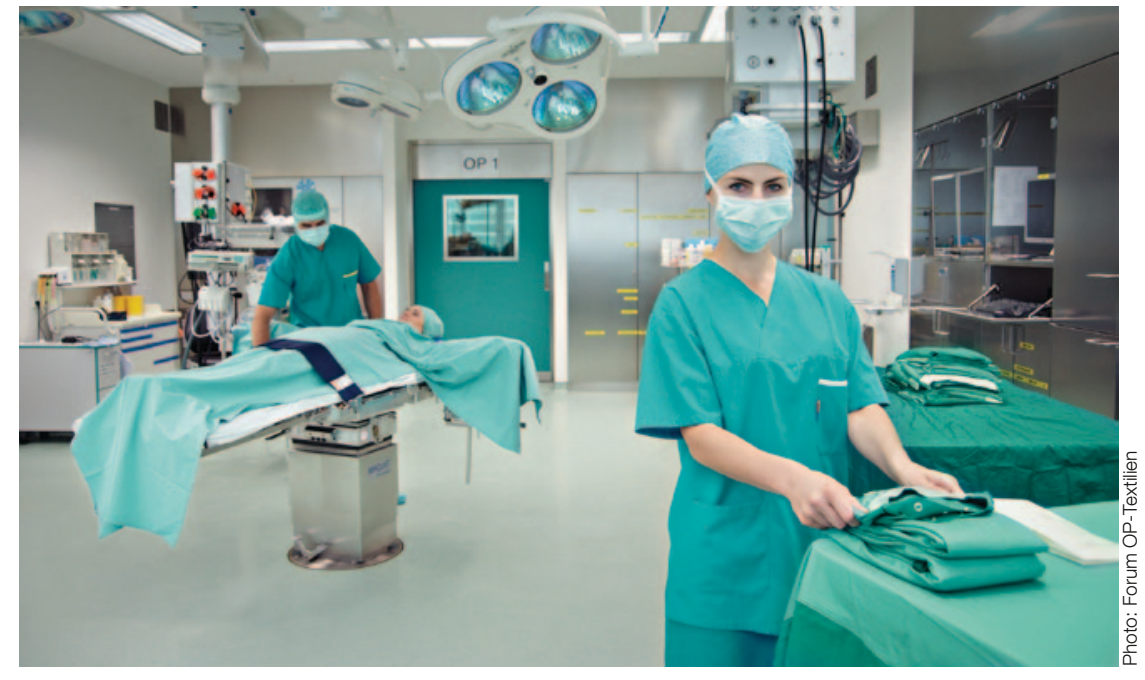

Ohne perfekte Arbeitsbedingungen werden im OP keine optimalen Leistungen erbracht.

Hertz, ärztlicher Leiter des Lorenz-Böhler Unfallkrankenhauses in Wien. Die deutlich bessere Atmungsaktivität der für Mehrwegtextilien verwendeten Materialien und daher der höhere Tragekomfort sind für den Unfallchirurgen wichtige Kriterien für die Entscheidung.

\section{Chirurgen und OP-Kleidung testen}

Mit einem neuen computergestützten arbeitspsychologischen Testsystem erfassten die Wissenschaftler des Hohenstein-Instituts das Konzentrationsvermögen von Chirurgen messtechnisch. Gemessen wurde, ob die Reaktionsgeschwindigkeit und die Fehlerhäufigkeit der Chirurgen durch das Tragen von Einweg- oder Mehrweg-Bekleidung unter OP-Bedingungen beeinflusst werden. Nach der Erledigung einer anspruchsvollen mikrochirurgischen Aufgabe in einer „Stressbox“, mussten die Probanden verschiedene Aufgaben erfüllen. Dabei erreichten die Studienteilnehmer mit Mehrwegbekleidung insgesamt bessere Ergebnisse hinsichtlich Reaktionszeit und Fehlerhäufigkeit als jene in Einwegkleidung.

Die SAFEC Studie, die auch österreichische Daten beinhaltete, zeigte, dass „bei qualifizierter Aufbereitung von wiederverwendbaren Medizinprodukten und bei entsprechendem Material- und Qualitätsmanagement die gestellten Anforderungen " hinsichtlich Schutz für Patienten, Anwender und Dritte, Hygiene, Trage- komfort und Handhabung ,erfüllt werden können", so der Konsensus. Demnach besitzen Mehrwegtextilien bezüglich Barriere-Eigenschaften, Partikelabgabe und mechanischer Festigkeit bei Nässe positive Eigenschaften und übertreffen die Vorgaben der Normen. Die verwendeten neuartigen Textilien erfüllen also alle Anforderungen und gewährleisten die Sicherheit der OP-Textilien.

Hinsichtlich der Kosten, die in den vergangenen Jahren immer mehr in den Blickpunkt bei der Produktauswahl gerückt sind, hält der Konsens fest, dass die tatsächlichen Kosten nicht ausschließlich vom Einkaufspreis abhängen, sondern auch vom Spital, von der Operationsfrequenz, den Patienten und den Aufbereitungsmöglichkeiten. Die Entscheidung sollte jedes Krankenhaus individuell nach den gegebenen Anforderungen treffen. Allerdings seien, so das Konsensus-Statement, bei einer Kostenrechnung, die alle bei einer OP benötigten Abdeckmaterialien berücksichtige, Mehrwegtextilien tendenziell wirtschaftlich attraktiver. Auch die Öko-Bilanz falle bei einer Gesamtrechnung mit Herstellung, Transport, Reinigung und Entsorgung günstiger für die Mehrwegprodukte aus.

Quelle: Pressekonferenz „Moderne OP-Textilien Patientensicherheit, Wirtschaftlichkeit \& Nachhaltigkeit" Veranstalter: Forum OP-Textilien, 7. 9. 2011 